\title{
Simple Optical Imaging of Nanoscale Features in Free-Standing Films
}

\author{
Peter J. Beltramo* and Jan Vermant \\ Department of Materials, ETH Zürich, Vladimir-Prelog-Weg 5, 8093 Zürich, Switzerland
}

\section{Supporting Information}

ABSTRACT: Measuring thicknesses in thin films with high spatial and temporal resolution is of prime importance for understanding the structure and dynamics in thin films and membranes. In the present work, we introduce fluorescenceinterferometry, a method that combines standard reflected light thin film interferometry with simultaneous fluorescence measurements. We apply this method to the thinning dynamics and phase separation in free-standing inverse phospholipid bilayer films. The measurements were carried out using a standard fluorescence microscope using multichannel imaging and yielded subnanometer resolution, which is applied to optically measure the discrete thickness variations across phase-separated membranes.

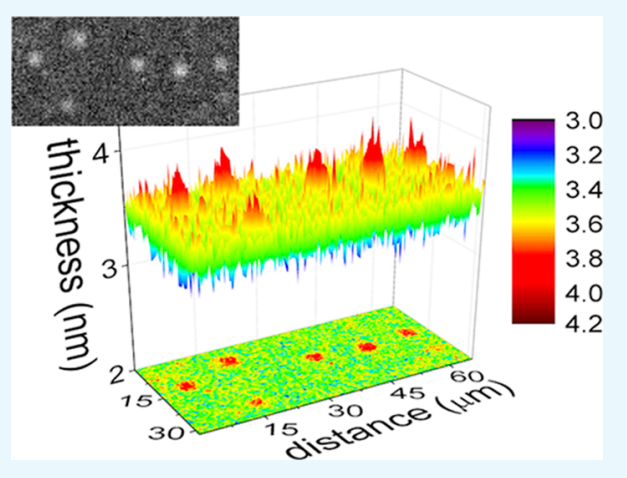

\section{INTRODUCTION}

The thickness of thin films and bilayer structures is paramount to understanding in many areas of technology and biology. For example, evaluating membrane heterogeneity and thickness fluctuations in in vitro model phospholipid bilayer systems can elucidate the fundamental underpinnings of biophysical processes, such as signaling and trafficking. This is known as the "lipid raft" hypothesis, where lipid micro- and nanodomains within the plane of the bilayer membrane laterally sort and organize certain proteins to orchestrate function. As a result, there has been extensive research into the phase separation of multicomponent membranes to understand the key forces and dynamic interactions at play. Specific questions to address in these systems include the following: (1) What is a temperature-composition phase diagram? (2) What are the relevant physical attributes, such as line tension, thickness and thickness heterogeneity, fluidity, and lipid molecular distribution, along this parameter space? (3) How do these morphological relationships affect function in model membranes and ultimately in in vivo systems? At the heart of these questions is the requirement to both visualize the domains and characterize their thicknesses; therefore, various techniques have been established to evaluate these parameters and address these questions. However, often a combination of techniques and experimental conditions is required for a comprehensive characterization of bilayer membranes.

For instance, fluorescence microscopy techniques have proven to be an invaluable tool to investigate membrane heterogeneity, revealing insights into lipid diffusion, ${ }^{2}$ lateral phase separation, ${ }^{3}$ line tension, ${ }^{4}$ membrane protein activity ${ }^{5}$ and sorting, ${ }^{6}$ and small-molecule translocation. ${ }^{7}$ However, the nanometer-scale thickness and the thickness variation between phase-separated domains make it difficult to measure the thickness of the bilayers using fluorescence. The $z$-resolutions of confocal and super-resolution microscopies are limited by the precision with which the point spread function can be deconvoluted. For example, confocal fluorescence microscopy could assess the degree of domain alignment between micronthick multilamellar membrane stacks, but to precisely evaluate thickness differences between domains, complementary experiments using atomic force microscopy (AFM) have been carried out. ${ }^{8}$ The increased spatial resolution of AFM comes at the expense of time resolution, which is sufficient for domain formation kinetics ${ }^{9-12}$ but too long for diffusion dynamics or line tension fluctuations. Ellipsometry offers a noncontact method to evaluate the phospholipid bilayer thickness with an improved temporal resolution and a larger field of view than AFM, ${ }^{13}$ but currently both methods are limited to supported bilayers, where extra precautions must be taken to ensure that the substrate does not influence the results, as opposed to freestanding membranes. Small-angle X-ray or neutron scattering techniques $^{14-17}$ (SAXS or SANS) provide a fine resolution of thickness variations in phase-separated phospholipid vesicles, but this requires accurate modeling of the scattering signal and gives an ensemble average of the properties without spatial resolution of individual domains. As a result, despite the multitude of methods addressing each problem individually, simultaneous measurements of the lipid bilayer thickness and optical monitoring of dynamic processes are not possible.

To measure the thickness of molecularly thin layers optically, interferometric methods are necessary. This is particularly challenging for free-standing (as opposed to supported) thin films. Reflection interference contrast microscopy (RICM) is a particularly useful tool to measure the thickness of model membranes adsorbed or proximate to a reflecting interface. ${ }^{18}$ Recently, it has also been extended to detect nanometer-scale

Received: July 15, 2016

Accepted: August 31, 2016

Published: September 12, 2016 
lipid phase separation without the need for fluorescent labels. ${ }^{19}$ For fully free-standing thin films, reflected light thin film interferometry is used and has the advantage of requiring simpler optical components. The main operating principle involves the incidence of monochromatic light orthogonally onto a planar micrometer- to nanometer-scale thickness film. By measuring the reflected light intensity and considering the values of the refractive index of the film and surroundings, the film thickness is calculated. This technique has been successfully and extensively applied to surfactant films studied in thin film balance experiments. Notable experimental and theoretical advances have been made, enabling new insights into the structure and dynamics of thin liquid films. Experimentally, the original glass capillary (Sheludko cell) ${ }^{20}$ and porous plate holders ${ }^{21,22}$ have been improved upon by either using microfluidic microfabrication ${ }^{23}$ or tapering the edges of a porous chip. ${ }^{24}$ These alternatives allow for symmetric drainage in the central orifice and the ability to apply a wider range of capillary pressures. A basic goal of these experiments is to link the interfacial material properties with film stability by measuring the equilibrium disjoining pressure versus film thickness isotherm or analyzing dynamic thinning processes. Sodium dodecyl sulfate (SDS) is commonly chosen as a model system, where above the critical micelle concentration $(\mathrm{cmc})$, an oscillatory disjoining pressure is measured because of the stepwise thinning based on the successive expulsion of micellar layers. ${ }^{23-27}$ Modeling of this phenomenon has determined the additive micelle structure interaction component of the disjoining pressure isotherm [in addition to the standard Derjaguin-Landau-Verwey-Overbeek (DLVO) contributions], ${ }^{28}$ and dynamic experiments and theory have linked the role of surface viscosity ${ }^{29}$ and surface viscoelasticity $^{30,31}$ on film stability. The versatility of this approach extends beyond ionic surfactants to include nonionic surfactants, ${ }^{32-34}$ polyelectrolytes, ${ }^{35,36}$ and also nonadsorbing particle suspensions. ${ }^{37}$ In some cases, for a more accurate determination of the film thickness, a three-layer model incorporating an independent measure of the refractive index and the thickness of the adsorbed surfactant layer was applied. ${ }^{38,39}$ Despite the progress made in the realm of surfactants, thin film balance experiments on aqueous dispersions of phospholipids ${ }^{40}$ and proteins ${ }^{41}$ are much rarer because of experimental challenges in microchip fabrication and pressure control; however, we have recently developed a system to overcome these issues. ${ }^{42}$

In this work, we address the need to simultaneously detect lipid phase separation and quantify bilayer thickness with high spatial and temporal resolutions in a noninvasive manner using a simple experimental setup. The technique relies on a combination of fluorescence and interferometric imaging using a solid-state light source and an upright microscope to measure the nanoscale thickness of free-standing, planar, inverse bilayer thin films. Inverse bilayer films, with phosphate headgroups in the interior and hydrocarbon tails on the exterior, are the opposite configuration of the bilayers composing cellular membranes. They contain the same lateral repulsion between headgroups and van der Waals attraction between the hydrocarbon tails, whereas they lack the tail interdigitation and interactions at the bilayer midplane. Therefore, studying the phase behavior and dynamics of inverse bilayers can offer insight into the importance and magnitude of different interactions on the overall interaction landscape that dictates cell membrane mechanics; such analogies are commonly made in the phospholipid monolayer literature. ${ }^{43}$ Here, using only a standard fluorescence microscope, we detect lipid phase separation and measure a thickness difference of $0.35 \mathrm{~nm}$ between fluid and gel phases in binary 1,2-dioleoyl-sn-glycero-3-phosphocholine (DOPC)/1,2-dipalmitoyl-sn-glycero-3-phosphocholine (DPPC) inverse bilayers.

\section{METHODS}

Materials. The phospholipids DOPC, DPPC, and 1,2dioleoyl-sn-glycero-3-phosphoethanolamine- $N$-(7-nitro-2-1,3benzoxadiazol-4-yl) ammonium salt (DOPE-NBD) were obtained from Avanti Polar Lipids. $\mathrm{NaCl}$ (99.99\%, metal basis) and $\mathrm{NaHCO}_{3}$ were obtained from Alfa Aesar. $\mathrm{CaCl}_{2}$ was purchased from Sigma-Aldrich. Ultrapure water was used (Milli-Q Merck-Millipore; resistivity, $<18.2 \mathrm{M} \Omega \mathrm{cm}$ ).

Sample Preparation. Lipid mixtures (DOPC/DPPC in a 3:2 molar ratio with $1 \mathrm{~mol} \mathrm{\%} \mathrm{fluorescent} \mathrm{DOPE-NBD)} \mathrm{in}$ chloroform are placed in a round-bottom flask and dried under a gentle stream of nitrogen. Any residual chloroform is removed by placing the sample under vacuum for at least $1 \mathrm{~h}$. The lipids are resuspended in an aqueous salt buffer $(150 \mathrm{mM} \mathrm{NaCl}, 2$ $\mathrm{mM} \mathrm{CaCl}$, and $0.2 \mathrm{mM} \mathrm{NaHCO}_{3}$ ) at a concentration of 0.5 $\mathrm{mg} / \mathrm{mL}$. The sample is tip-sonicated (Hielscher UP400S, 30\% pulse and $75 \%$ amplitude) after the addition of the buffer until the solution is clear. The lipid solutions are used within a week of preparation.

Experimental Setup. We have recently developed a thin film balance apparatus to create large-area planar phospholipid bilayers with tension control. ${ }^{42}$ Here, we briefly overview how our setup is used to investigate inverse bilayers in air. The thin film balance is composed of a custom designed microfluidic bikewheel chip (Micronit Microfluidics) with a $1 \mathrm{~mm}$ diameter orifice at the center. Before use, the bikewheel microchips are made hydrophilic. The chips are cleaned in a saturated solution of $\mathrm{NaOH}$ in ethanol in an ultrasonication bath for $20 \mathrm{~min}$ before being thoroughly rinsed with water. The chip is glued to a titanium holder using UHU two-component epoxy and placed in a temperature-controlled pressure chamber. The capillary pressure in the microchip is controlled by a differential pressure transducer (Baratron 120AD) interfaced with a syringe pump (Harvard Apparatus PHD Ultra CP).

The microchip is loaded with a preheated lipid/buffer mixture before inserting it into the chamber at the desired temperature $\left(43{ }^{\circ} \mathrm{C}\right)$ and connecting the pressure control tubing. A thick film of aqueous lipid solution is formed within the bikewheel annulus and allowed to drain by increasing the capillary pressure. A fast drainage rate is used to induce dimple formation and to calibrate the reflected light intensity, whereas a slower controlled drainage is used to encourage the formation of a single inverse phospholipid bilayer film at the center of the orifice.

For observing inverse bilayer formation and measuring lipid domain appearance, the sample is imaged on an upright microscope (Nikon Eclipse FN1) with a 10× long-workingdistance objective and a Hamamatsu ORCA-Flash4.0 CMOS camera. A multi-solid-state LED light source (Nikon Spectra-X) is cycled between wavelengths $\lambda=508$ and $470 \mathrm{~nm}$. A quadband filterset (Nikon FRE17055) matches the spectral excitation and emission of the fluorescent lipid, allowing for fluorescence microscopy using $\lambda=470 \mathrm{~nm}$, while also transmitting $\lambda=508 \mathrm{~nm}$ for reflection imaging. Two important aspects of this setup are (1) the use of a 16 bit camera, which is necessary to resolve the small changes in intensity in both 


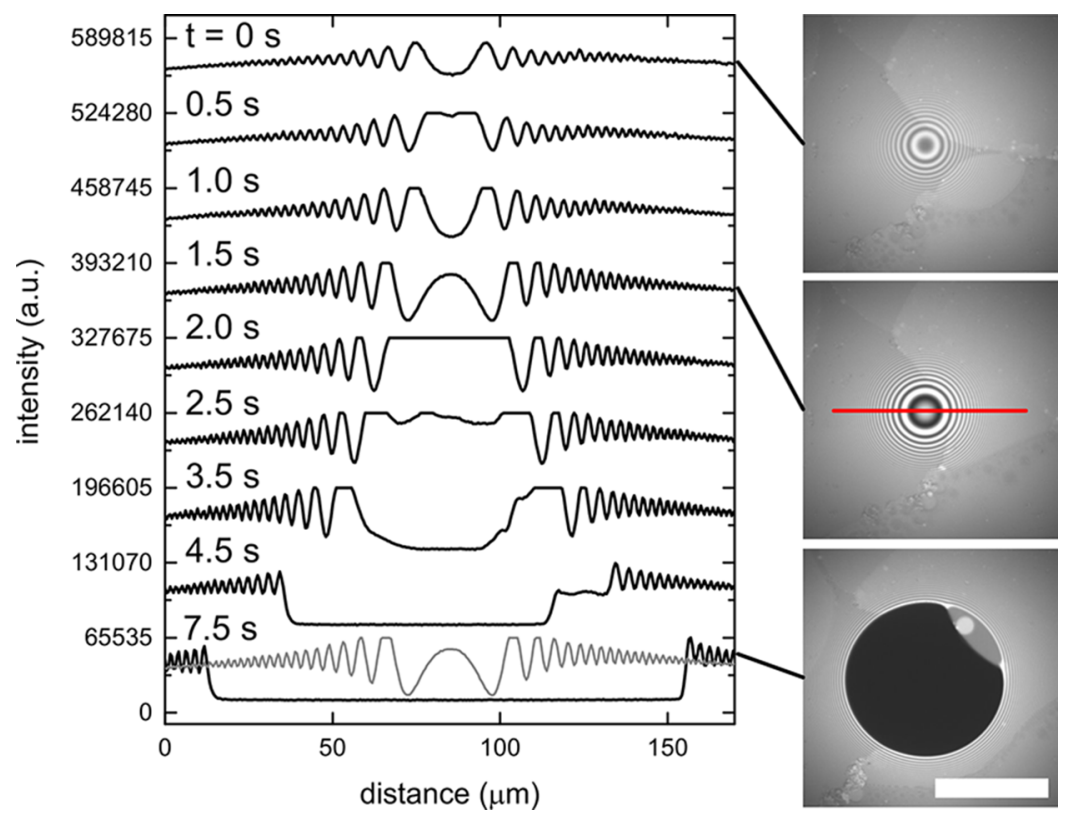

Figure 1. Reflected light intensity profile along the center of the film during fast drainage of an aqueous phospholipid suspension to determine $I_{\max }$ and $I_{\min }$ using the standard procedure. The profiles are offset by a constant value (65 535), and the $t=1.5 \mathrm{~s}$ profile is replotted for comparison with the final profile. A single inverse bilayer phospholipid film forms over the course of $8 \mathrm{~s}$, as shown in the images on the right. The scale bar represents $100 \mu \mathrm{m}$.

fluorescence and reflectance signals and (2) high-speed triggering between the light source and the camera, which enable multichannel imaging at a maximum frame rate of 100/ $N$ fps, where $N$ is the number of channels required.

\section{RESULTS AND DISCUSSION}

For a thin liquid film surrounded by two bulk phases of equal refractive index (in our case, air), the reflected light intensity $I$ from the incidence of monochromatic light orthogonal to the film is related to the film thickness by

$$
h_{i}=\left(\frac{\lambda}{2 \pi n_{\mathrm{f}}}\right) \arcsin \left(\sqrt{\frac{\Delta_{i}}{1+4 R\left(1-\Delta_{i}\right) /(1-R)^{2}}}\right)
$$

where $\Delta=\left(I_{i}-I_{\min }\right) /\left(I_{\max }-I_{\min }\right)$ is the scaled intensity, $\lambda$ is the wavelength of light, $R=\left[\left(n_{\mathrm{f}}-n_{\text {air }}\right) /\left(n_{\mathrm{f}}+n_{\text {air }}\right)\right]^{2}$ is the Fresnel reflectance coefficient, $n_{\mathrm{f}}$ and $n_{\text {air }}$ are the refractive indices of the film and air, respectively, and $i$ denotes different areas of the thin film. ${ }^{20}$ Crucial to accurate determination of the film thickness is the determination of $I_{\max }$ and $I_{\min }$, which are the maximum and minimum reflected light intensities corresponding to the positive and negative reflected light interference occurring at film thicknesses equal to integer values of $\lambda / 4$, and using an appropriate value for the thin film refractive index. In thin film studies of surfactant films, $I_{\max }$ and $I_{\min }$ are traditionally determined by rapidly draining the film and monitoring the intensity at the fringes that appear. ${ }^{23,44}$ As the film drains through various orders of $\lambda / 4 \mathrm{~nm}$ thicknesses, the repeated detection of maximum and minimum values of the reflected light intensity gives confidence in the values of $I_{\max }$ and $I_{\min }$ used. A more subtle increase in the disjoining pressure is then used to realize stable films. The reflected light intensity from the stable thin films that form when performing a disjoining pressure isotherm measurement is between these two values, and the film thickness ranges from $>100$ to $10 \mathrm{~nm}$. The film thickness measured using eq 1 is termed an "equivalent" thickness because the bulk value of the solution refractive index is used for $n_{\mathrm{f}}$. This is justified because of the minimal thickness of the adsorbed surfactant layers in comparison to the overall film thickness. Corrections can also be made for a refractive index difference between the bulk solution and the surfactant, which requires an independent measure of the thickness and the refractive index of the adsorbed surfactant layer. ${ }^{39,44,45} \mathrm{We}$ discuss the ramifications of using different values for the film refractive index in our results below.

We have found that the resolution of reflective light interferometry is insufficient for aqueous films of lipids because the final film that forms is within the noise of $I_{\min }$ often being slightly darker than any of the fringes that form during rapid drainage (Figure 1). This prevents an accurate measurement of the thickness. Note that in Figure 1, we have increased the incident light intensity to emphasize the failure to determine $I_{\min }$, with the signal becoming saturated where $I_{\max }$ would be found. Motivated by this failure, we have developed a way to combine information from fluorescence microscopy and the reflected light intensity signal in order to accurately determine inverse bilayer lipid film thicknesses. For a film region with uniform thickness, eq 1 has three unknowns: $h, I_{\max }$ and $I_{\min }$. In essence, we use the information from the fluorescence signal to develop a series of equations relating the thickness of different areas of the film to determine $I_{\max }$ and $I_{\min }$ using the reflected light intensity from those areas alone. This is particularly notable in that we can now optically measure the subnanometer changes in bilayer thicknesses that occur due to lipid phase separation.

One of the additional challenges we have encountered while studying inverse lipid bilayers in a thin film balance is the tendency for the lipids to form lamellar aggregates in solution. However, this is used to our advantage in the current method because the thicknesses of these multilayer stacks are quantized by the molecular dimensions of the phospholipids. Figure 2 shows the thin film formed after rapid drainage from a mixture 

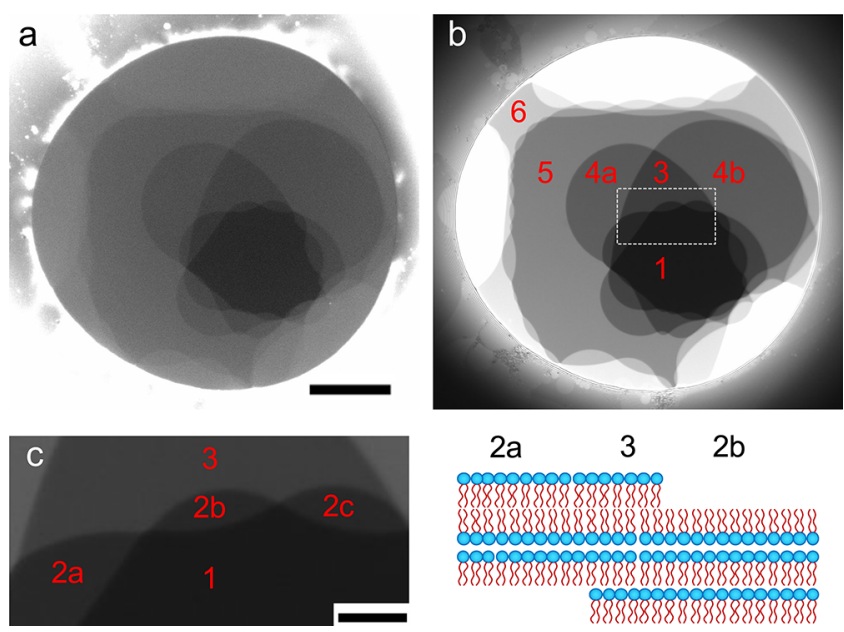

Figure 2. Fluorescence (a) and reflectance (b) microscopy images after thinning of a phospholipid solution to form a thin film with lamellar aggregates. (c) Magnified view of the reflectance of an area of the film showing the overlap in lamellar areas and a schematic to explain the overlap of regions $2 \mathrm{a}$ and $2 \mathrm{~b}$ to form region $\mathrm{c}$. Numbered regions are explained in the text and correspond to Table 1 . The scale bar represents $100 \mu \mathrm{m}$ for (a) and (b) and $20 \mu \mathrm{m}$ for (c).

of lipids (DOPC/DPPC in a 3:2 molar ratio with $1 \mathrm{~mol} \%$ fluorescent DOPE-NBD at $43{ }^{\circ} \mathrm{C}$ ) that shows a series of lamellar aggregates. The fluorescence signal (Figure 2a) and the reflected light intensity (Figure $2 b, c$ ) both exhibit the same pattern across the film. From this, it is obvious that the different areas of fluorescence/reflected light intensity have different film thicknesses. Furthermore, from the fluorescence, we can infer relations between the different thicknesses based on the fact that these thickness differences arise from a varying number of lamellar layers. Table 1 gives the fluorescence intensity for the

Table 1. Average Quantities of the Fluorescence and Reflected Light Intensities for the Regions Indicated in Figure 2, with the Calculated Film Thickness Based on the Method Explained in the Text

\begin{tabular}{lcccccc} 
& \multicolumn{2}{c}{ fluorescence } & & \multicolumn{3}{c}{ interferometry } \\
\cline { 2 - 3 } \cline { 5 - 6 } area & $I-I_{\mathrm{bkg}}$ & $\frac{I-I_{\mathrm{bkg}}}{I_{1}-I_{\mathrm{bkg}}}$ & & intensity & $h(\mathrm{~nm})$ & $h / h_{1}$ \\
\hline 1 & $243 \pm 67$ & 1 & & $10875 \pm 178$ & $3.91 \pm 0.12$ & 1 \\
$2 \mathrm{a}$ & $357 \pm 70$ & 1.47 & & $14229 \pm 197$ & $5.73 \pm 0.09$ & 1.47 \\
$2 \mathrm{~b}$ & $348 \pm 70$ & 1.43 & & $14101 \pm 200$ & $5.67 \pm 0.09$ & 1.45 \\
$2 \mathrm{c}$ & $356 \pm 68$ & 1.47 & & $14136 \pm 190$ & $5.69 \pm 0.09$ & 1.45 \\
3 & $460 \pm 70$ & 1.90 & & $19259 \pm 232$ & $7.62 \pm 0.08$ & 1.97 \\
$4 \mathrm{a}$ & $572 \pm 73$ & 2.36 & & $26385 \pm 374$ & $9.75 \pm 0.10$ & 2.52 \\
$4 \mathrm{~b}$ & $597 \pm 73$ & 2.46 & & $26031 \pm 358$ & $9.66 \pm 0.10$ & 2.50 \\
5 & $715 \pm 90$ & 2.95 & & $33522 \pm 308$ & $11.65 \pm 0.07$ & 2.98 \\
6 & $899 \pm 130$ & 3.70 & $53399 \pm 518$ & $15.66 \pm 0.09$ & 4.00 \\
\hline
\end{tabular}

different areas of the film indicated in Figure 2. We observe immediately that the fluorescence intensity is quantized in logical increments assuming the darkest (thinnest) layer, area 1, is a single bilayer. Note, in particular, the curvature at the borders of area 2 highlighted in Figure $2 \mathrm{c}$ and the observation that all parts of area 2 have the same reflected light intensity. The border between areas $2 \mathrm{a}$ and 1 is continuous with that of area $3 /$ area $2 b$, and finally the area $2 c$ /area 1 border. This indicates that areas $2 \mathrm{a}$ and $2 \mathrm{c}$ are part of the same additional phospholipid layer on one side of the inverse bilayer constituting area 1 . The independent continuity of the border between areas $2 a$ and $3,2 b / 1$, and $2 c / 3$ indicate that area $2 b$ arises from the same additional thickness, but occurring on the opposite side of the single inverse bilayer in area 1. Lastly, where these additional lamellar layers overlap, the reflected light intensity increases as a result of the thicker film. This is illustrated schematically in Figure $2 \mathrm{~d}$, and an analogous observation can be made for layers $4 a / b$.

This observation is quantitatively confirmed with the fluorescence signal. We assume that area 1 is a single inverse bilayer based on the fact that this layer is the darkest, on par with the darkest intensity detected from the rapid drainage of the film, and we never observe a thinner blacker film. Based on the hydrophilic phospholipid headgroups and hydrophobic hydrocarbon tails, we assume that an "inverse" bilayer with a morphology opposite to that of a traditional cell membrane is formed. Area 2 has 1.5 times more fluorescence than area 1 , implying that area 2 is this single bilayer with an additional monolayer of lipid (Table 1). Likewise, the fluorescence of area 3 is double that of area 1 , indicating two inverse bilayers, which makes sense based on our observations above about the overlap of the different regions. The quantization continues through thicker layers, with the fluorescence from area 6 being approximately 4 times that of area 1 (four inverse bilayers). Even thicker lamellar stacks can be observed in the fluorescence; however, eventually the reflected light intensity saturates.

Returning to eq 1 , we now have several options to deduce a set of equations to solve for $I_{\max }$ and $I_{\min }$, and ultimately the film thickness. Equation 1 can be written independently for each region of the film specified in Figure 2, but they share the same $I_{\max }$ and $I_{\min }$ values. Therefore, at minimum, two independent equations relating three values of $h_{i}$ are needed to establish a solvable set of five equations (three forms of eq 1 and two height relations) and five unknowns (three thicknesses and intensity minima and maxima). One option, chosen for simplicity in the subsequent calculations, relates the thickness of area 3 with that of area $1, h_{3}=2 h_{1}$, and area 6 with that of area $3, h_{6}=2 h_{3}$.

Now, we are in a position to solve for $I_{\max }$ and $I_{\min }$. Substituting the expressions for eq 1 to the height relations, we have

$$
\begin{aligned}
& h_{3}=2 h_{1} \\
& \left(\frac{\lambda}{2 \pi n_{\mathrm{f}}}\right) \arcsin \left(\sqrt{\frac{\Delta_{3}}{1+4 R\left(1-\Delta_{3}\right) /(1-R)^{2}}}\right) \\
& \quad=2\left(\frac{\lambda}{2 \pi n_{\mathrm{f}}}\right) \arcsin \left(\sqrt{\frac{\Delta_{1}}{1+4 R\left(1-\Delta_{1}\right) /(1-R)^{2}}}\right)
\end{aligned}
$$

$$
\arcsin \left(\Psi_{3}\right)=2 \arcsin \left(\Psi_{1}\right)
$$

where we have substituted $\Psi_{i}$ for the square root term for compactness. Taking the sine of both sides gives

$$
\Psi_{3}=\sin \left(2 \arcsin \left(\Psi_{1}\right)\right)
$$

which can be reduced using the double angle rule to

$$
\Psi_{3}=2 \Psi_{1} \cos \left(\arcsin \left(\Psi_{1}\right)\right)
$$

and finally 

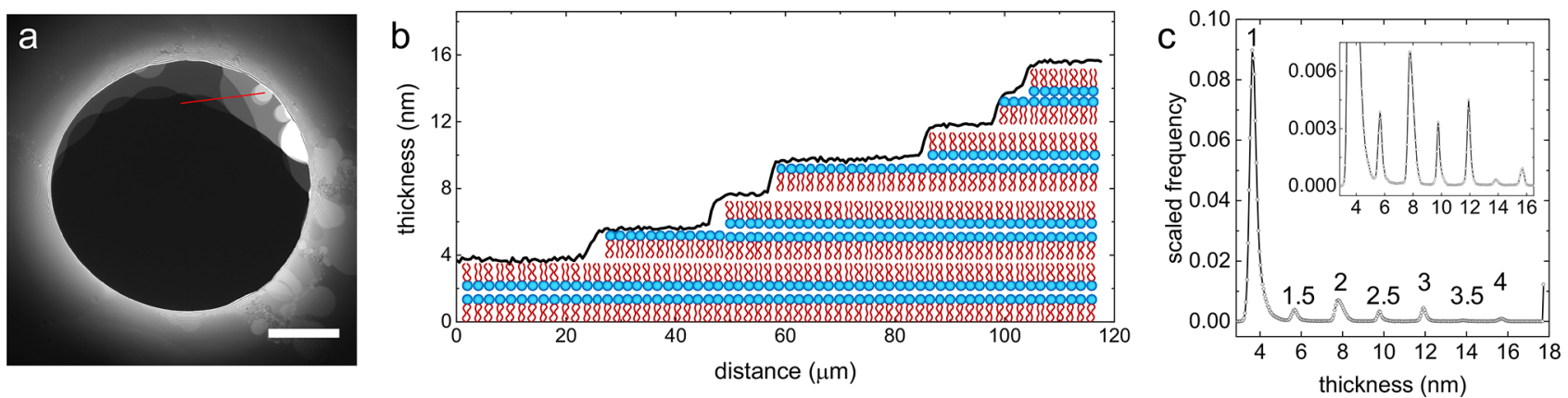

Figure 3. (a) Reflected light microscopy image of an inverse bilayer film with some lamellar areas in the upper portion. The scale bar represents 100 $\mu \mathrm{m}$. (b) Thickness profile along the red line in the image, where more than four lamellar lipid layers are identified in some cases. The line is the height profile calculated using the $I_{\max }$ and $I_{\min }$ values deduced from the procedure in the text, and underneath is a cartoon of the different lamellar layers. (c) Histogram of the thickness distribution within the film showing the stepwise multilayering, with the nominal $h / h_{1}$ values given above the peaks. The reflected light intensity saturated for approximately $1 \%$ of the film, indicating greater than 4 lamellar layers (last data point).

$$
\Psi_{3}=2 \Psi_{1} \sqrt{1-\Psi_{1}^{2}}
$$

Analogously for $h_{6}=2 h_{3}$, we have

$$
\Psi_{6}=2 \Psi_{3} \sqrt{1-\Psi_{3}^{2}}
$$

The only unknowns in eqs 7 and 8 are $I_{\max }$ and $I_{\min }$ because the unscaled reflected light intensity for each region $\left(I_{i=1,3,6}\right)$ is known and given in column 4 of Table 1 . We solve this system of equations numerically and find that $I_{\max }=526834$ and $I_{\min }=$ 7945. Note that to enhance the contrast between the reflected light intensity of the different lamellar layers in the film we have increased the incident light intensity, which causes the fitted value for $I_{\max }$ to be above the maximum possibly detected by the 16-bit grayscale CMOS camera used.

Using the solved values for $I_{\max }$ and $I_{\min }$, we apply eq 1 to all areas of the film to determine the film thickness values reported in column 5 of Table 1 . As expected, the values for the thinnest layers are of sub-10 nanometer scale, with the thickness of a single inverse bilayer being approximately $4 \mathrm{~nm}$. This agrees well with the expected thickness of a lipid bilayer deduced from scattering and AFM methods. ${ }^{14,15,46}$ The height ratios assumed from the fluorescence intensity are carried over to the film thickness. Importantly, no information from areas 2, 4, or 5 was used in this derivation, yet the film thickness measured for these areas agrees with the height ratios given in their respective fluorescence intensities. To further check the success of this method, we derived another set of equations relating the thicknesses, using $h_{3}=2 h_{1}$, as before, and also $h_{3}=2 h_{2}-h_{1}$. The latter relation is unique in that it actually does not rely on the fluorescence signal because it can be implied based on the continuity of the area borders as explained above. The trigonometric details for this set of equations are provided in the Supporting Information. In addition to eq 7 (and instead of eq 8 ), we now have

$$
\Psi_{3}=2 \Psi_{2}\left(\sqrt{1-\Psi_{2}^{2}}\right)\left(\sqrt{1-\Psi_{1}^{2}}\right)-\left(1-2 \Psi_{2}^{2}\right) \Psi_{1}
$$

using $h_{3}=2 h_{2}-h_{1}$. This set can also be solved to give similar values of $I_{\max }$ and $I_{\min }$, resulting in thicknesses in great agreement with the values found earlier. In this case, no information from area 4, 5, or 6 of the film was used in this deduction, yet accurate thickness ratios were obtained.

In simplifying and solving the equations, we have assumed a uniform film refractive index which is equal to that of DPPC in the liquid expanded phase measured using ellipsometry, $n_{\mathrm{f}}=$
1.455 . $^{47,48}$ This is chosen because at the expected thicknesses, the phospholipids are the main component of the film and there are many measurements on DPPC as opposed to DOPC. Although we do not know the tilt angle of the phospholipid chains in the film, anisotropy in the refractive index is minimal. The values for $I_{\max }$ and $I_{\min }$ do not change appreciably even if the refractive index of water is used to solve eqs 7 and 8 . We also evaluated the thickness based on a different assumption: using the refractive index of the phosphate headgroup/water interior as $n_{\mathrm{f}}=1.40^{13}$ to calculate the equivalent thickness and correcting for the film thickness using the three-layer method. $^{44,45}$ This presents an additional complication that an assumed value of the phospholipid hydrocarbon layer thickness must be used, which we do not know in situ and could vary from that measured by ellipsometry/reflectivity using monolayers or bilayer vesicles because of variations in packing and chain tilt. Nevertheless, using this approach and a varying hydrocarbon layer of 1.1-1.4 nm thickness resulted in an overall film thickness in the range of $3.73-3.64 \mathrm{~nm}$, approximately $0.2 \mathrm{~nm}$ less than that reported in Table 1 . To minimize the number of assumptions necessary, we feel justified in using a uniform value of the refractive index based on the phospholipids, but potentially, simultaneous determination of other parameters could improve the accuracy. Additional sources of variability in the measurement include the bandwidth of the incident light $(17 \mathrm{~nm})$, noise in the camera detection, and intrinsic thickness fluctuations in the bilayer. These factors combine to cause the greatest error in measurement at the smallest film thicknesses. Considering the bandwidth of the incident light, the uncertainty in the actual film thickness is $1.7 \%$ of its magnitude, which corresponds to $\pm 0.07 \mathrm{~nm}$ for the single inverse bilayer. Camera noise is practically negligible; using the same exposure, the dark counts of the camera have an average intensity of $102 \pm 4$. These optics are already excellent for measuring the inverse bilayer film thickness and phase separation, as explained below; however, performing the measurement with a longer-wavelength incident light source and narrowing its bandwidth are strategies to increase measurement precision.

With values for $I_{\max }$ and $I_{\min }$ in hand, the two- or threedimensional film thickness can now be determined accurately. To illustrate this, Figure 3a shows the reflected light intensity of a thin film formed from the same lipid mixture with smaller lamellar areas in the top right of the film. A linescan across this area reveals the sharp steps in the film thicknesses, which span 

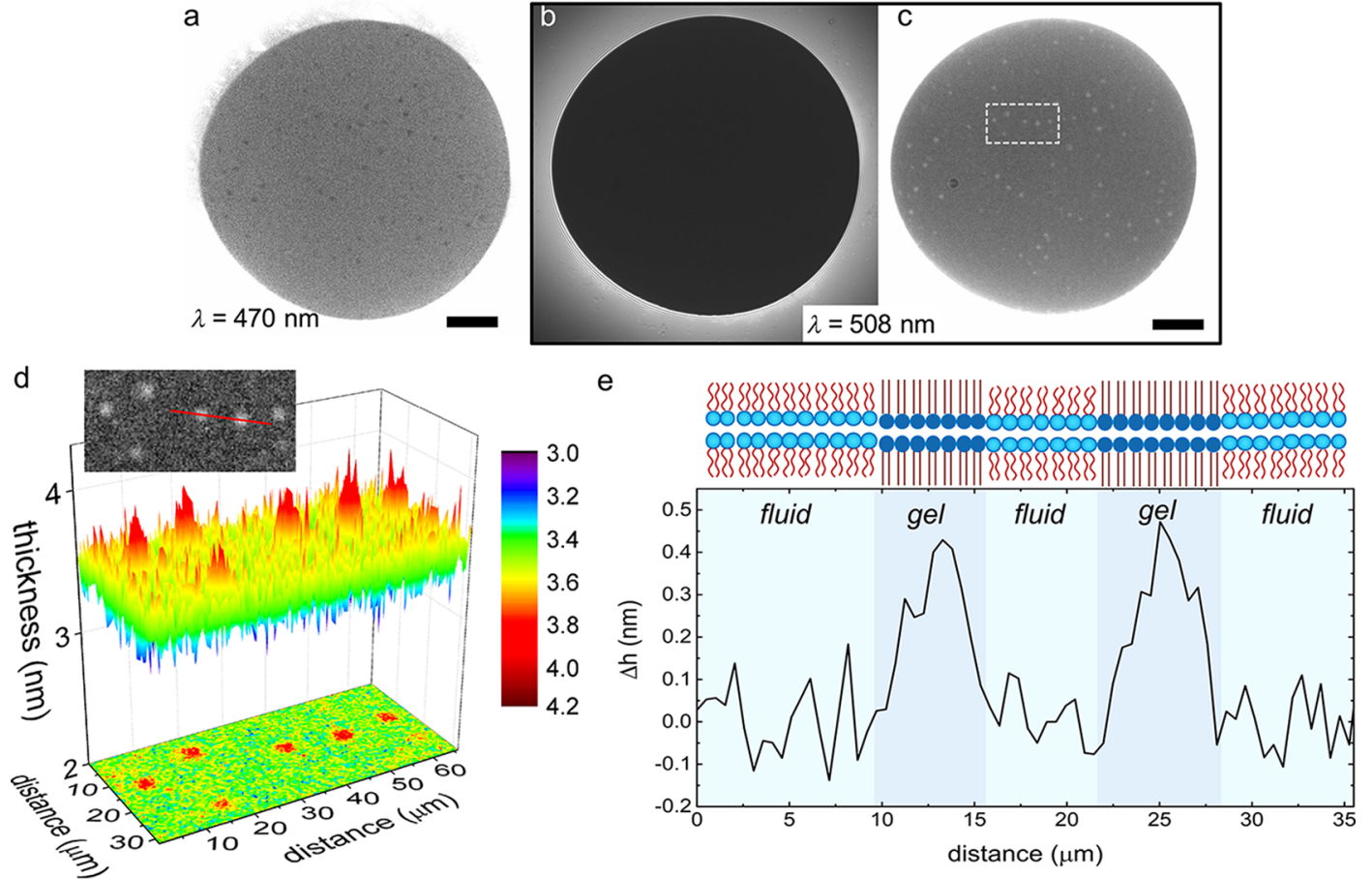

Figure 4. (a) Fluorescence image showing the appearance of dark domains when the temperature is cooled to $37^{\circ} \mathrm{C}$. (b) Corresponding reflectance microscopy image appears to show a single black film when unscaled, but (c) upon adjusting the contrast bright spots are evident in positions corresponding to the dark domains in the fluorescence image. (d) Magnified view of a section of the film with the corresponding three-dimensional thickness map. The thicker gel phase (red) protrudes from the majority fluid phase (yellow/green). (e) Thickness profile of the black lipid film across two of the domains in (d), with an illustration of the gel and fluid phases. The scale bar for panel $(\mathrm{a}-\mathrm{c})$ represents $50 \mu \mathrm{m}$.

from a single inverse lipid bilayer $3.9 \mathrm{~nm}$ thick to four stacked inverse bilayers $15.6 \mathrm{~nm}$ thick (Figure $3 \mathrm{~b}$ ). Note that in our schematic, we cannot assess the geometry of the regions that contain half of a bilayer (an additional monolayer) or how the edges are stabilized. We do note that the lamellar areas do not form from stepwise thinning of micelles, as in typical surfactant films above the $\mathrm{cmc}$ such as SDS, ${ }^{23-27}$ but rather are stable layers that move around the film as it expands or contracts. This feature is detrimental to performing disjoining pressure isotherm measurements because the boundary conditions of the film are not symmetric, but it does of course enable us to determine the film thickness and potentially investigate the ramifications of different solutions or lipid conditions on the morphology of such multilayer stacks. The portions of the film with an odd number of lipid monolayers must have an energetically unfavorable configuration at some point in the stack (for instance, with the phospholipid headgroups exposed to the atmosphere instead of the hydrophobic tails). This is also evident in the discretized distribution of thicknesses observed in the entire film, as shown in Figure 3c. Interestingly, the film is more likely to have a thickness with an integer number of bilayers than the "half" bilayer immediately preceding it (i.e., $7.8 \mathrm{~nm}$ is more common than $5.7 \mathrm{~nm}, 11.9 \mathrm{~nm}$ than $9.8 \mathrm{~nm}$, and $15.6 \mathrm{~nm}$ than $13.8 \mathrm{~nm}$ ). We suspect that this is due in part to the unfavorable configurations of those noninteger bilayer regions.

We have combined interferometry with logical and reasonable assumptions on the relationship between different lamellar areas of the film to determine the sub-10-nanometerscale thicknesses of lipid thin films. This result can now be leveraged to directly detect and measure the thickness differences between coexisting lipid domains in phaseseparating lipid mixtures. Specifically in our case, fluid-gel phase separation has been extensively documented in the binary DPPC/DOPC phospholipid bilayer system. ${ }^{49-53}$ As mentioned in the introduction, the inverse bilayers maintain the lateral interactions of membrane bilayers while possessing different midplane interactions; therefore, although we use the same terminology, the ramifications of these geometric differences on the precise boundaries and characteristics of the phase diagram remain an open area of research.

To form a uniform film, a smaller pressure gradient is applied to thin the liquid, which results in a single inverse lipid bilayer film without any lamellar layers. Doing this at high temperatures results in a single-phase bilayer with homogeneous fluorescence intensity. In Figure 4, the lipid film has been cooled below its phase transition temperature to $37{ }^{\circ} \mathrm{C}$. As mentioned earlier, it is standard practice to detect the presence of lipid domains by the exclusion of fluorophore from the more fluid domain, and we observe this in the fluorescence signal of Figure 4a. However, the fluorescence signal has no information on the relative thickness difference between the two phases because it is dictated by the concentration of fluorescent lipid molecules partitioning between the two phases. The condensed gel phase consists of phospholipids with rigid hydrocarbon tails, making these domains in the bilayer thicker than the fluid phase.

On first glance, the reflected light intensity channel appears featureless (Figure 4b), but when increasing the contrast (by linearly rescaling the grayscale between 8500 and 13000 ) and inspecting the reflected light signal closely, we see changes in intensity in precisely the same areas of the film where domains are forming according to the fluorescence signal (Figure 4c). Thus, reflected light microscopy is capable of distinguishing between coexisting domains in the thin lipid bilayers, and the increased intensity of the domain regions is expected because 
they are thicker due to the condensed phase lipids. Given the small temperature difference and the minimal variation of the refractive index with temperature, we use the $I_{\max }$ and $I_{\min }$ values obtained above to measure the film thickness in an area of the film where domains are present (Figure 4d). Six domains with radii $\sim 2 \mu \mathrm{m}$ are easily distinguished. The brighter/red areas correspond to the gel phase and have an average thickness of $3.81 \pm 0.11 \mathrm{~nm}$, whereas the majority fluid phase has an average thickness of $3.47 \pm 0.13 \mathrm{~nm}$. Figure $4 \mathrm{e}$ shows the thickness profile (with the average thickness of the fluid phase subtracted) along two of the domains to more clearly indicate the overall thickness difference between the two phases of $\Delta h=$ $0.34 \pm 0.16 \mathrm{~nm}$. The preceding was calculated based on a laterally uniform film refractive index. Ellipsometric measurements indicate that the refractive index of DPPC increases from 1.455 to 1.503 when moving from the liquid expanded to the gel phase at the water/air monolayer interface, ${ }^{47}$ and considering an increase in the bilayer refractive index of this magnitude, the thickness difference between the domains decreases slightly to $0.17 \mathrm{~nm}$.

Our measurements are in agreement or on the lower end of the range when compared with the results obtained from scattering or AFM on membrane bilayers. García-Sáez et al. ${ }^{11}$ found height differences ranging from 0.2 to $1.3 \mathrm{~nm}$, depending on composition, using AFM of ternary lipid mixtures. Giocondi et al. ${ }^{9}$ used AFM on 3:1 DOPC/DPPC-supported lipid bilayers and found a height difference of $1.2 \mathrm{~nm}$ at $23{ }^{\circ} \mathrm{C}$. Similarly, SAXS of DOPC/DPPC/cholesterol multilamellar vesicles yielded thicknesses of $4.6 \mathrm{~nm}$ for the liquid ordered phase and $3.9 \mathrm{~nm}$ for the liquid disordered phase, ${ }^{16}$ whereas SANS of quaternary lipid mixtures yielded thickness differences between 0.6 and $1 \mathrm{~nm} .{ }^{17}$ The advantage of the present technique rests on the real-time formation and observation with a simple microscope, which allows the domains to be imaged directly, their dynamics tracked, and their shape fluctuations deduced. If the optics of a given experiment remains constant and $I_{\max }$ and $I_{\min }$ are already calibrated, this technique could also be potentially applied to measure lipid raft properties without the use of fluorescent tagging.

\section{CONCLUSIONS}

Detection of coexisting lipid domains is important to analyze processes that mimic "lipid raft"-like processes. Optical fluorescence measurements are ideal for visualizing individual domains, their shape, fluctuations or translational dynamics, whereas scattering (or AFM) methods are needed to measure the thickness variations between phases. We have combined thin film reflected light microscopy with fluorescence microscopy to measure the thicknesses of inverse bilayer films. The temporal and spatial resolutions of the approach are limited only by the camera and the microscope objective used, respectively. Using this method, we are able to detect and quantify the thickness differences in phase-separated domains of inverse phospholipid bilayers, which are in agreement with that found using standard bilayers. This microscopy-based method shows promise now to investigate the dynamics of such domains in conjunction with their height fluctuations. In addition, the appearance of multilamellar areas in the thin film allows for domain registration across the lamella to be measured. Lastly, although our current focus of research is lipid bilayers, this thin film balance-based technique can be equally extended to study the properties of other molecular thin films, such as nanoparticles, copolymers, or surfactants.

\section{ASSOCIATED CONTENT}

\section{Supporting Information}

The Supporting Information is available free of charge on the ACS Publications website at DOI: 10.1021/acsomega.6b00125.

Detailed derivation of equations to solve intensity minima/maxima (PDF)

\section{AUTHOR INFORMATION}

\section{Corresponding Author}

*E-mail: peter.beltramo@mat.ethz.ch.

\section{Notes}

The authors declare no competing financial interest.

\section{ACKNOWLEDGMENTS}

Funding was provided by ETH Zürich Grant no. ETH-03 15-2 and SNSF Grant no. 200021_165974.

\section{REFERENCES}

(1) Lingwood, D.; Simons, K. Lipid Rafts As a Membrane-Organizing Principle. Science 2009, 327, 46-50.

(2) Stanich, C. A.; Honerkamp-Smith, A. R.; Putzel, G. G.; Warth, C. S.; Lamprecht, A. K.; Mandal, P.; Mann, E.; Hua, T.-A. D.; Keller, S. L. Coarsening Dynamics of Domains in Lipid Membranes. Biophys. J. 2013, 105, 444-454.

(3) Veatch, S. L.; Keller, S. L. Separation of Liquid Phases in Giant Vesicles of Ternary Mixtures of Phospholipids and Cholesterol. Biophys. J. 2003, 85, 3074-3083.

(4) Honerkamp-Smith, A. R.; Cicuta, P.; Collins, M. D.; Veatch, S. L.; den Nijs, M.; Schick, M.; Keller, S. L. Line Tensions, Correlation Lengths, and Critical Exponents in Lipid Membranes Near Critical Points. Biophys. J. 2008, 95, 236-246.

(5) Huang, S.; Romero-Ruiz, M.; Castell, O. K.; Bayley, H.; Wallace, M. I. High-throughput optical sensing of nucleic acids in a nanopore array. Nat. Nanotechnol. 2015, 10, 986-991.

(6) Aimon, S.; Callan-Jones, A.; Berthaud, A.; Pinot, M.; Toombes, G. E. S.; Bassereau, P. Membrane Shape Modulates Transmembrane Protein Distribution. Dev. Cell 2014, 28, 212-218.

(7) Dezi, M.; Di Cicco, A.; Bassereau, P.; Lévy, D. Detergentmediated incorporation of transmembrane proteins in giant unilamellar vesicles with controlled physiological contents. Proc. Natl. Acad. Sci. U.S.A. 2013, 110, 7276-7281.

(8) Tayebi, L.; Ma, Y.; Vashaee, D.; Chen, G.; Sinha, S. K.; Parikh, A. $\mathrm{N}$. Long-range interlayer alignment of intralayer domains in stacked lipid bilayers. Nat. Mater. 2012, 11, 1074-1080.

(9) Giocondi, M.-C.; Vié, V.; Lesniewska, E.; Milhiet, P.-E.; ZinkeAllmang, M.; Le Grimellec, C. Phase Topology and Growth of Single Domains in Lipid Bilayers. Langmuir 2001, 17, 1653-1659.

(10) Johnston, L. J. Nanoscale Imaging of Domains in Supported Lipid Membranes. Langmuir 2007, 23, 5886-5895.

(11) García-Sáez, A. J.; Chiantia, S.; Schwille, P. Effect of Line Tension on the Lateral Organization of Lipid Membranes. J. Biol. Chem. 2007, 282, 33537-33544.

(12) Connell, S. D.; Heath, G.; Olmsted, P. D.; Kisil, A. Critical point fluctuations in supported lipid membranes. Faraday Discuss. 2013, 161, 91-111.

(13) Howland, M. C.; Szmodis, A. W.; Sanii, B.; Parikh, A. N. Characterization of Physical Properties of Supported Phospholipid Membranes Using Imaging Ellipsometry at Optical Wavelengths. Biophys. J. 2007, 92, 1306-1317.

(14) Kučerka, N.; Nieh, M.-P.; Katsaras, J. Fluid phase lipid areas and bilayer thicknesses of commonly used phosphatidylcholines as a function of temperature. Biochim. Biophys. Acta, Biomembr. 2011, 1808, 2761-2771.

(15) Marquardt, D.; Heberle, F. A.; Nickels, J. D.; Pabst, G.; Katsaras, $\mathrm{J}$. On scattered waves and lipid domains: Detecting membrane rafts with X-rays and neutrons. Soft Matter 2015, 11, 9055-9072. 
(16) Heftberger, P.; Kollmitzer, B.; Rieder, A. A.; Amenitsch, H.; Pabst, G. In Situ Determination of Structure and Fluctuations of Coexisting Fluid Membrane Domains. Biophys. J. 2015, 108, 854-862.

(17) Heberle, F. A.; Petruzielo, R. S.; Pan, J.; Drazba, P.; Kučerka, N.; Standaert, R. F.; Feigenson, G. W.; Katsaras, J. Bilayer Thickness Mismatch Controls Domain Size in Model Membranes. J. Am. Chem. Soc. 2013, 135, 6853-6859.

(18) Rädler, J.; Sackmann, E. Imaging optical thicknesses and separation distances of phospholipid vesicles at solid surfaces. J. Phys. II 1993, 3, 727-748.

(19) de Wit, G.; Danial, J. S. H.; Kukura, P.; Wallace, M. I. Dynamic label-free imaging of lipid nanodomains. Proc. Natl. Acad. Sci. U.S.A. 2015, 112, 12299-12303.

(20) Sheludko, A. Thin liquid films. Adv. Colloid Interface Sci. 1967, 1, 391-464.

(21) Mysels, K. J. Soap Films and Some Problems in Surface and Colloid Chemistry1. J. Phys. Chem. 1964, 68, 3441-3448.

(22) Mysels, K. J.; Jones, M. N. Direct measurement of the variation of double-layer repulsion with distance. Discuss. Faraday Soc. 1966, 42, $42-50$.

(23) Pereira, L. G. C.; Johansson, C.; Blanch, H. W.; Radke, C. J. A bike-wheel microcell for measurement of thin-film forces. Colloids Surf., A 2001, 186, 103-111.

(24) Bergeron, V.; Radke, C. J. Equilibrium Measurements of Oscillatory Disjoining Pressures in Aqueous Foam Films. Langmuir 1992, 8, 3020-3026.

(25) Nikolov, A. D.; Wasan, D. T. Ordered Micelle Structuring in Thin-Films Formed from Anionic Surfactant Solutions .1. Experimental. J. Colloid Interface Sci. 1989, 133, 1-12.

(26) Anachkov, S. E.; Danov, K. D.; Basheva, E. S.; Kralchevsky, P. A.; Ananthapadmanabhan, K. P. Determination of the aggregation number and charge of ionic surfactant micelles from the stepwise thinning of foam films. Adv. Colloid Interface Sci. 2012, 183, 55-67.

(27) Zhang, Y.; Sharma, V. Domain expansion dynamics in stratifying foam films: Experiments. Soft Matter 2015, 11, 4408-4417.

(28) Nikolov, A. D.; Kralchevsky, P. A.; Ivanov, I. B.; Wasan, D. T. Ordered micelle structuring in thin films formed from anionic surfactant solutions: II. Model development. J. Colloid Interface Sci. 1989, 133, 13-22.

(29) Ivanov, I. B.; Dimitrov, D. S. Hydrodynamics of thin liquid films. Colloid Polym. Sci. 1974, 252, 982-990.

(30) Sonin, A. A.; Bonfillon, A.; Langevin, D. Role of surface elasticity in the drainage of soap films. Phys. Rev. Lett. 1993, 71, 2342-2345.

(31) Langevin, D.; Sonin, A. A. Thinning of soap films. Adv. Colloid Interface Sci. 1994, 51, 1-27.

(32) Danov, K. D.; Ivanov, I. B.; Ananthapadmanabhan, K. P.; Lips, A. Disjoining pressure of thin films stabilized by nonionic surfactants. Adv. Colloid Interface Sci. 2006, 128-130, 185-215.

(33) Exerowa, D.; Platikanov, D. Thin liquid films from aqueous solutions of non-ionic polymeric surfactants. Adv. Colloid Interface Sci. 2009, 147-148, 74-87.

(34) Christov, N. C.; Danov, K. D.; Zeng, Y.; Kralchevsky, P. A.; von Klitzing, R. Oscillatory Structural Forces Due to Nonionic Surfactant Micelles: Data by Colloidal-Probe AFM vs Theory. Langmuir 2009, 26, 915-923.

(35) Asnacios, A.; Espert, A.; Colin, A.; Langevin, D. Structural Forces in Thin Films Made from Polyelectrolyte Solutions. Phys. Rev. Lett. 1997, 78, 4974-4977.

(36) Beltrán, C. M.; Langevin, D. Stratification Kinetics of Polyelectrolyte Solutions Confined in Thin Films. Phys. Rev. Lett. 2005, 94, 217803.

(37) Wasan, D. T.; Nikolov, A. D.; Kralchevsky, P. A.; Ivanov, I. B. Universality in film stratification due to colloid crystal formation. Colloids Surf. 1992, 67, 139-145.

(38) Stubenrauch, C.; Schlarmann, J.; Strey, R. A disjoining pressure study of $n$-dodecyl-beta-D-maltoside foam films. Phys. Chem. Chem. Phys. 2002, 4, 4504-4513.
(39) Bergeron, V.; Waltermo, A.; Claesson, P. M. Disjoining pressure measurements for foam films stabilized by a nonionic sugar-based surfactant. Langmuir 1996, 12, 1336-1342.

(40) Todorov, R.; Cohen, R.; Exerowa, D. Surface forces in foam films from DPPC and lung surfactant phospholipid fraction. Colloids Surf., A 2007, 310, 32-38.

(41) Pereira, L. G. C.; Johansson, C.; Radke, C. J.; Blanch, H. W. Surface forces and drainage kinetics of protein-stabilized aqueous films. Langmuir 2003, 19, 7503-7513.

(42) Beltramo, P. J.; Van Hooghten, R; Vermant, J. Millimeter-area, free standing, phospholipid bilayers. Soft Matter 2016, 12, 4324-4331.

(43) Stefaniu, C.; Brezesinski, G.; Möhwald, H. Langmuir monolayers as models to study processes at membrane surfaces. Adv. Colloid Interface Sci. 2014, 208, 197-213.

(44) Claesson, P. M.; Ederth, T.; Bergeron, V.; Rutland, M. W. Techniques for measuring surface forces. Adv. Colloid Interface Sci. 1996, 67, 119-183.

(45) Stubenrauch, C.; von Klitzing, R. Disjoining pressure in thin liquid foam and emulsion films-new concepts and perspectives. J. Phys.: Condens. Matter 2003, 15, R1197-R1232.

(46) Leonenko, Z. V.; Finot, E.; Ma, H.; Dahms, T. E. S.; Cramb, D. $\mathrm{T}$. Investigation of Temperature-Induced Phase Transitions in DOPC and DPPC Phospholipid Bilayers Using Temperature-Controlled Scanning Force Microscopy. Biophys. J. 2004, 86, 3783-3793.

(47) Ducharme, D.; Max, J. J.; Salesse, C.; Leblanc, R. M. Ellipsometric study of the physical states of phosphatidylcholines at the air-water interface. J. Phys. Chem. 1990, 94, 1925-1932.

(48) Thoma, M.; Schwendler, M.; Baltes, H.; Helm, C. A.; Pfohl, T.; Riegler, H.; Möhwald, H. Ellipsometry and X-ray Reflectivity Studies on Monolayers of Phosphatidylethanolamine and Phosphatidylcholine in Contact with $n$-Dodecane, $n$-Hexadecane, and Bicyclohexyl. Langmuir 1996, 12, 1722-1728.

(49) Lentz, B. R.; Barenholz, Y.; Thompson, T. E. Fluorescence depolarization studies of phase transitions and fluidity in phospholipid bilayers. 2. Two-component phosphatidylcholine liposomes. Biochemistry 1976, 15, 4529-4537.

(50) Parasassi, T.; Gratton, E.; Yu, W. M.; Wilson, P.; Levi, M. Twophoton fluorescence microscopy of laurdan generalized polarization domains in model and natural membranes. Biophys. J. 1997, 72, 24132429.

(51) de Almeida, R. F. M.; Borst, J.; Fedorov, A.; Prieto, M.; Visser, A. J. W. G. Complexity of Lipid Domains and Rafts in Giant Unilamellar Vesicles Revealed by Combining Imaging and Microscopic and Macroscopic Time-Resolved Fluorescence. Biophys. J. 2007, 93, 539-553.

(52) Mills, T. T.; Huang, J.; Feigenson, G. W.; Nagle, J. F. Effects of cholesterol and unsaturated DOPC lipid on chain packing of saturated gel-phase DPPC bilayers. Gen. Physiol. Biophys. 2009, 28, 126.

(53) Suga, K.; Umakoshi, H. Detection of Nanosized Ordered Domains in DOPC/DPPC and DOPC/Ch Binary Lipid Mixture Systems of Large Unilamellar Vesicles Using a TEMPO Quenching Method. Langmuir 2013, 29, 4830-4838. 